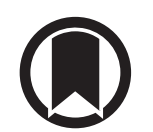

CrossMark

\title{
Chronic thromboembolic disease following pulmonary embolism: time for a fresh look at old clot
}

\author{
To the Editor:
}

The recently updated European Society of Cardiology/European Respiratory Society guidelines for acute pulmonary embolism (PE) underline the importance of appropriate long-term management of $\mathrm{PE}$ sequelae in the era of extended anticoagulation [1]. Chronic thromboembolic disease (CTED) is one of several conditions contributing to breathlessness in this setting, i.e. persistent pulmonary vascular obstruction on imaging, with no evidence of pulmonary hypertension at rest. CTED is increasingly encountered in pulmonary vascular disease clinics following acute PE, and its diagnosis relies heavily on careful exclusion of other conditions that may contribute to symptoms. Given the need for careful follow-up after PE, in this paper we highlight two important points relating to the diagnosis and management of CTED: firstly, the location and extent of chronic pulmonary artery thrombus on imaging and secondly, the lack of evidence to support long-term anticoagulation in CTED, as opposed to chronic thromboembolic pulmonary hypertension (CTEPH).

The haemodynamic definition of CTED includes patients with evidence of pulmonary vascular obstruction and a mean pulmonary artery pressure $<25 \mathrm{mmHg}$ at rest assessed by right heart catheterisation [2]. In line with CTEPH, the diagnosis of CTED usually requires at least 3 months anticoagulation prior to the diagnostic work-up. The original description of CTED as a novel diagnosis, distinct from CTEPH, included highly selected symptomatic patients referred for pulmonary endarterectomy (PEA) on account of a large thrombotic burden on computed tomography (CT) pulmonary angiography (CTPA), mild right ventricle (RV) enlargement on echocardiography, but no pulmonary hypertension at rest [3]. The same research group later reported clinical improvement in terms of World Health Organization functional class and quality of life scores following surgery in a more extended CTED cohort [4]. Recently, the diagnostic definition of CTED has transformed to encompass breathless patients following acute PE who, despite anticoagulation, demonstrate persistent areas of perfusion/ventilation mismatch on nuclear scintigraphy, irrespective of the presence or location of thrombus on CT.

In broadening the clinical definition to include patients with distal vascular obstruction, more patients are being diagnosed with CTED. Pulmonary vascular resistance (PVR) is usually minimally affected by anatomical thrombus distribution. However, impedance to pulmonary forward flow is not well-captured by PVR and may be markedly different between patients with proximal versus distant lesions, with a greater effect on RV afterload arising from proximal pulmonary vascular obstruction $[5,6]$. Discrimination between proximal thrombus on CT and more distal obstruction on scintigraphy may, therefore, be important in defining the haemodynamic effect of CTED, and its impact on the RV and symptoms.

Secondly, anticoagulation in CTEPH is prescribed under a class 1 recommendation, and recourse to extended anticoagulation in CTED is extrapolated from this guideline [2]. However, when a diagnosis of CTED is made after an index PE associated with a major transient risk factor, such as immobilisation related to surgery, clinical uncertainty arises and the decision to pursue extended anticoagulation may not automatically apply. "Post-PE syndrome" has recently been introduced to describe permanent changes in pulmonary artery flow, gas exchange and/or cardiac function in the breathless patient following PE. While this entity links symptoms to abnormal gas exchange and RV dysfunction, definitive anticoagulation

@ERSpublications

Chronic thromboembolic disease diagnosed following acute pulmonary embolism warrants refinement of its definition and further evaluation of optimal anticoagulation strategies http://bit.ly/38SQXc0

Cite this article as: McCabe C, Dimopoulos K, Pitcher A, et al. Chronic thromboembolic disease following pulmonary embolism: time for a fresh look at old clot. Eur Respir J 2020; 55: 1901934 [https://doi.org/10.1183/13993003.01934-2019]. 
recommendations are lacking [7]. We have learnt from the ELOPE study that post-PE breathlessness is unrelated to both clot burden and choice of anticoagulation [8]; however, low levels of pulmonary vascular obstruction in this study leave unanswered questions around optimal anticoagulation strategies in patients with larger thrombotic burden after PE.

In our view, the current diagnostic landscape appears too broad to allow emergence of meaningful clinical guidance on CTED management. To streamline this approach, we suggest a diagnostic label of CTED be reserved for symptomatic patients with chronic proximal thrombus on CTPA in whom, in the alternative context of pulmonary hypertension, clinicians would consider surgical referral for PEA. In our experience, such patients more frequently exhibit mild RV dilatation on echocardiography than those without proximal thrombus on CTPA and are more likely to benefit from prolonged anticoagulation irrespective of PE risk factors. In contrast, symptomatic patients with isolated perfusion defects on nuclear scintigraphy at 3-6 months following PE with no proximal thrombotic burden on CTPA require prospective evaluation within anticoagulation studies, grouped by the presence of transient risk factors at the time of PE and/or risk factors for CTEPH. We therefore propose an algorithm formulated from our clinical experience (figure 1), in which a diagnosis of CTED becomes the preserve of pulmonary hypertension expert centres where exercise haemodynamic evaluation, PEA referral or balloon pulmonary angioplasty can be offered $[9,10]$. This algorithm, currently untested in management studies, simplifies the differentiation between CTED and post-PE breathlessness at 3-6 months follow-up by sole reliance on imaging without the need for cardiopulmonary exercise testing which carries greater variability in interpretation. With few individuals eligible for PEA, anticoagulation remains the only medical therapy that may be offered to most CTED patients. Criteria for the identification of patients who are likely to benefit from extended anticoagulation, versus those who may safely discontinue treatment with no detriment to their quality of

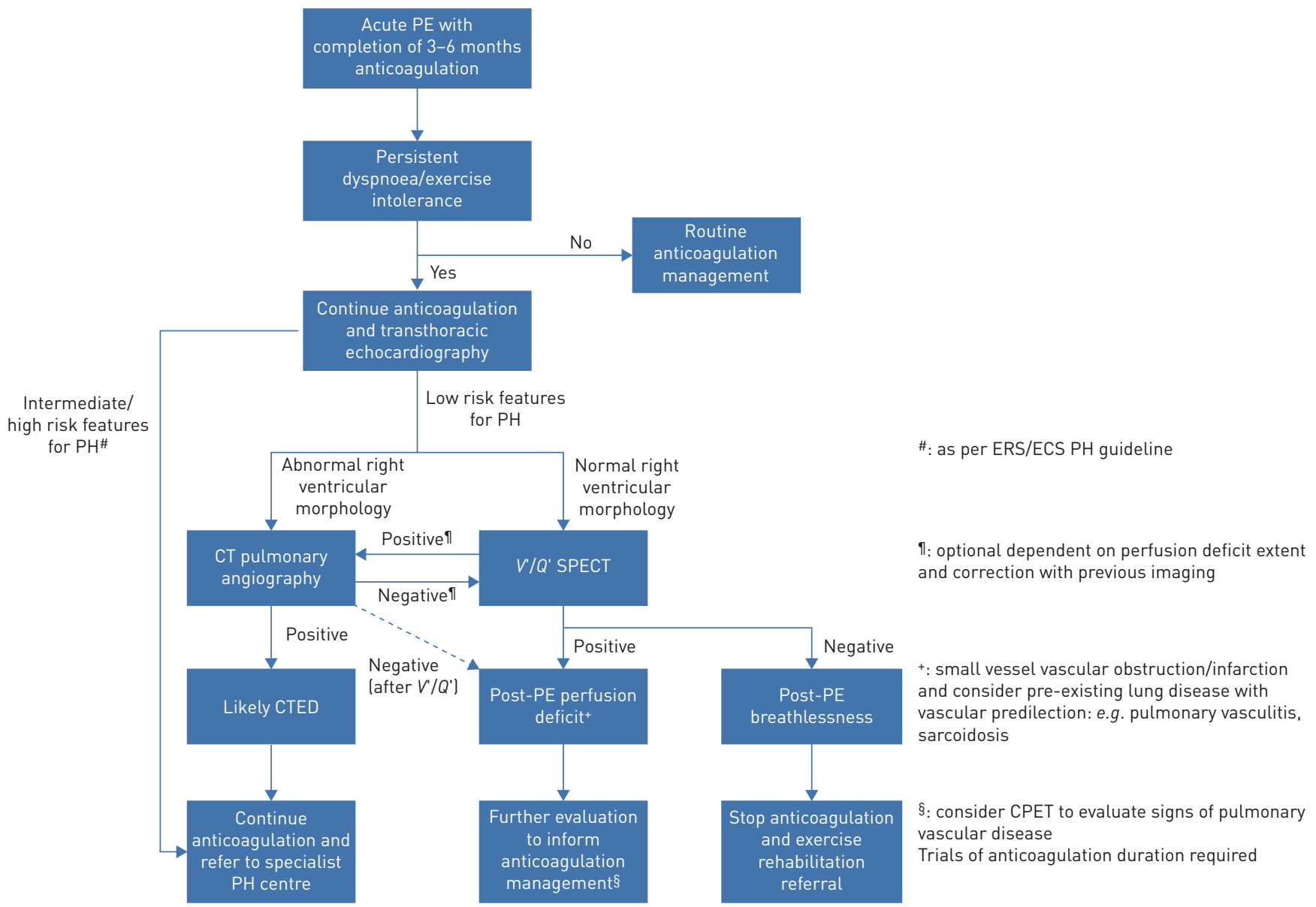

FIGURE 1 Proposed algorithm for patients with persistent dyspnoea following acute pulmonary embolism (PE). CPET: cardiopulmonary exercise testing; CTED: chronic thromboembolic disease; $V^{\prime} / Q^{\prime}$ SPECT: ventilation/perfusion single-photon emission computed tomography; PH: pulmonary hypertension. 
life or risk of PE recurrence, will only emerge with a more robust definition of CTED in this diverse patient population.

Colm McCabe ${ }^{1,2}$, Konstantinos Dimopoulos ${ }^{1,2}$, Alex Pitcher ${ }^{3}$, Elizabeth Orchard $^{3}$, Laura C. Price ${ }^{1,2}$, Aleksander Kempny ${ }^{1,2}$ and S. John Wort ${ }^{1,2}$

${ }^{1}$ Dept of Pulmonary Hypertension, Royal Brompton Hospital, London, UK. ${ }^{2}$ National Heart and Lung Institute, Imperial College, London, UK. ${ }^{3}$ Dept of Cardiology, Oxford University Hospital NHS Foundation Trust, Oxford, UK.

Correspondence: Colm McCabe, Royal Brompton Hospital, Pulmonary Hypertension Service, Sydney Street, London SW3 6NP, UK. E-mail: c.mccabe2@rbht.nhs.uk

Received: 1 Oct 2019 | Accepted after revision: 24 Jan 2020

Conflict of interest: None declared.

\section{References}

1 Konstantinides SV, Meyer G, Becattini C, et al. 2019 ESC Guidelines for the diagnosis and management of acute pulmonary embolism developed in collaboration with the European Respiratory Society (ERS). Eur Respir J 2019; 54: 1901647.

2 Galie N, Humbert M, Vachiery JL, et al. 2015 ESC/ERS Guidelines for the diagnosis and treatment of pulmonary hypertension: The Joint Task Force for the Diagnosis and Treatment of Pulmonary Hypertension of the European Society of Cardiology (ESC) and the European Respiratory Society (ERS. Eur Heart J; 2016: 67-119.

3 McCabe C, White PA, Hoole SP, et al. Right ventricular dysfunction in chronic thromboembolic obstruction of the pulmonary artery: a pressure-volume study using the conductance catheter. J Appl Physiol 2014; 116: 355-363.

4 Taboada D, Pepke-Zaba J, Jenkins DP, et al. Outcome of pulmonary endarterectomy in symptomatic chronic thromboembolic disease. Eur Respir J 2014; 44: 1635-1645.

5 Pagnamenta A, Vanderpool R, Brimioulle S, et al. Proximal pulmonary arterial obstruction decreases the time constant of the pulmonary circulation and increases right ventricular afterload. J Appl Physiol 2013; 114: 1586-1592.

6 Su J, Hughes $\mathrm{AD}$, Simonsen $\mathrm{U}$, et al. Impact of pulmonary endarterectomy on pulmonary arterial wave propagation and reservoir function. Am J Physiol Heart Circ Physiol 2019; 317: H505-HH16.

7 Klok FA, van der Hulle T, den Exter PL, et al. The post-PE syndrome: a new concept for chronic complications of pulmonary embolism. Blood Rev 2014; 28: 221-226.

8 Kahn SR, Hirsch AM, Akaberi A, et al. Functional and exercise limitations after a first episode of pulmonary embolism: results of the ELOPE prospective cohort study. Chest 2017; 151: 1058-1068.

9 Inami $\mathrm{T}$, Kataoka $\mathrm{M}$, Kikuchi $\mathrm{H}$, et al. Balloon pulmonary angioplasty for symptomatic chronic thromboembolic disease without pulmonary hypertension at rest. Int J Cardiol 2019; 289: 116-118.

10 Swietlik EM, Ruggiero A, Fletcher AJ, et al. Limitations of resting haemodynamics in chronic thromboembolic disease without pulmonary hypertension. Eur Respir J 2019; 53: 1801787.

\section{Chronic thromboembolic disease following pulmonary embolism: more work ahead}

From the author:

The comment by C. McCabe and colleagues provides thoughtful insights into the late sequalae of pulmonary embolism. The authors' views and suggestions, based on their clinical experience and expertise, may prove helpful in the ongoing process of reliably (and meaningfully) distinguishing chronic

@ERSpublications

From acute to chronic pulmonary embolism http://bit.ly/3893ubd

Cite this article as: Konstantinides SV. Chronic thromboembolic disease following pulmonary embolism: more work ahead. Eur Respir J 2020; 55: 2000229 [https://doi.org/10.1183/13993003.002292020]. 
thromboembolic pulmonary hypertension (CTEPH) from chronic thromboembolic disease (CTED) without pulmonary hypertension at rest.

A key novelty of the 2019 European Society of Cardiology (ESC) guidelines for the diagnosis and management of acute pulmonary embolism, developed in collaboration with the European Respiratory Society (ERS), is the conceptual evolution in the way we view and manage thrombosis and pulmonary embolism [1]. It is now widely acknowledged that pulmonary embolism is, at least in the majority of cases, not a brief disease state that may occasionally result in the patient's death in the acute phase, while those who survive to hospital discharge will live happily ever after or will "only" need to concentrate on their underlying disease. On the contrary, we have now finally recognised the amplitude of the public health problem that results from the large proportion of patients complaining of chronic, persistent symptoms or functional limitation after PE $[2,3]$. Of these patients, only few will ultimately be diagnosed with a severe, life-threatening chronic pulmonary obstructive vasculopathy, either with (CTEPH) or without pulmonary hypertension at rest (CTED). In other words, pulmonary embolism has now officially become a "chronic" disease, or at least one that needs chronic attention and follow-up. Endorsing this change of paradigm, the task force of the 2019 pulmonary embolism guidelines set the focus on developing a new algorithm and updated recommendations to guide clinicians through the multiple challenges and potential pitfalls of post-pulmonary embolism care [1]. Of course, the guidelines still summarise the "essentials" of CTEPH and, to a lesser extent, CTED, i.e. those principles of pathophysiology, diagnosis and treatment that every physician caring for a patient with pulmonary embolism needs to know. On the other hand, the task force unanimously decided that it was beyond the scope of these pulmonary embolism guidelines to interfere with recent and ongoing discussions which attempt to better characterise the groups of pulmonary hypertension, notably CTEPH and CTED [4]. This task is left to the ESC/ERS guidelines on pulmonary hypertension [5], and to their future update.

C. McCabe and colleagues point to a number of unresolved issues concerning the diagnosis and actually the very definition of CTED, and its differential diagnosis from CTEPH. They also touch upon a controversial topic, namely the need for anticoagulation and, if there is one, the appropriate anticoagulant for patients with CTED. In this regard, the results of a recent retrospective analysis cast doubt on the efficacy and safety of non-vitamin K antagonist oral anticoagulants (NOACs) in the setting of CTEPH and perhaps CTED in the broader sense [6]. Clearly, this is only a hypothesis and a caveat at the moment, but it highlights the need for prospective research in a field which the NOACs have largely seized from the vitamin $\mathrm{K}$ antagonists without a battle.

C. McCabe and colleagues propose a sophisticated, quite complex algorithm for diagnosing the severe late sequelae of pulmonary embolism, notably CTEPH and CTED. This algorithm has not been formally validated and therefore remains a working hypothesis at present. In any case, one cannot agree more with the authors that the diagnosis of CTED (and CTEPH) should be the preserve of pulmonary hypertension expert centres where exercise haemodynamic evaluation, pulmonary endarterectomy referral or balloon pulmonary angioplasty can be offered. But what the general practitioner does need to know is which patients, out of all survivors of acute pulmonary embolism, to refer to these centres. This is where the 2019 pulmonary embolism guidelines have done their best to provide helpful guidance. It will ultimately be upon the medical community and the guideline users, the physicians themselves, to judge if they succeeded.

Stavros V. Konstantinides $\oplus^{1,2}$

${ }^{1}$ Center for Thrombosis and Hemostasis, University Medical Center of the Johannes Gutenberg University, Mainz, Germany. ${ }^{2}$ Dept of Cardiology, Democritus University of Thrace, Alexandroupolis, Greece.

Correspondence: Stavros V. Konstantinides, Centre for Thrombosis and Haemostasis, University Medical Centre Mainz, Langenbeckstrasse 1, Bldg. 403, 55131 Mainz, Germany. E-mail: stavros.konstantinides@unimedizin-mainz.de

Received: 3 Feb 2020 | Accepted after revision: 3 Feb 2020

Conflict of interest: S.V. Konstantinides reports grants and non-financial support from Bayer AG; grants and personal fees from Boehringer Ingelheim, Actelion, Daiichi-Sankyo, Biocompatibles Group UK and MSD, personal fees from Bayer AG and Pfizer-Bristol-Myers Squibb, all outside the submitted work.

Support statement: The work of Stavros Konstantinides was supported by the German Federal Ministry of Education and Research (BMBF 01EO1003). The author is responsible for the contents of this publication.

\section{References}

1 Konstantinides SV, Meyer G, Becattini C, et al. 2019 ESC Guidelines for the diagnosis and management of acute pulmonary embolism developed in collaboration with the European Respiratory Society (ERS). Eur Respir J 2019; 54: 1901647. 
2 Kahn SR, Hirsch AM, Akaberi A, et al. Functional and exercise limitations after a first episode of pulmonary embolism: results of the ELOPE prospective cohort study. Chest 2017; 151: 1058-1068.

3 Kahn SR, Akaberi A, Granton JT, et al. Quality of life, dyspnea, and functional exercise capacity following a first episode of pulmonary embolism: results of the ELOPE cohort study. Am J Med 2017; 130: 990-990.

4 Galie N, McLaughlin VV, Rubin LJ, et al. An overview of the 6th World Symposium on Pulmonary Hypertension. Eur Respir J 2019; 53: 1802148.

5 Galie N, Humbert M, Vachiery JL, et al. 2015 ESC/ERS Guidelines for the diagnosis and treatment of pulmonary hypertension: The Joint Task Force for the Diagnosis and Treatment of Pulmonary Hypertension of the European Society of Cardiology (ESC) and the European Respiratory Society (ERS). Eur Heart J 2016; 37: 67-119.

6 Bunclark K, Newnham M, Chiu YD, et al. A multicenter study of anticoagulation in operable chronic thromboembolic pulmonary hypertension. J Thromb Haemost 2020; 18: 114-122. 